\title{
The Association between Chronic Periodontitis and Chronic Obstructive Pulmonary Disease - A Review
}

\section{Dongxue Zhang and Zuomin Wang*}

Department of Stomatology, Beijing Chaoyang Hospital, Capital Medical University, China

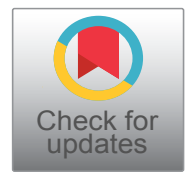

*Corresponding author: Dr. Zuomin Wang, Department of Stomatology, Beijing Chaoyang Hospital, Capital Medical University, Beijing, China

\begin{abstract}
Periodontitis is an inflammatory disease, which is characterized by the destruction of the tooth-supporting structures resulting from an overreaction of the inflammatory process. Chronic obstructive pulmonary disease (COPD) is a prime pulmonary disease characterized by a limitation in the airflow.

The possible relationship between chronic periodontitis and COPD has become an increasing focus of research. The aspiration of oral pathogens into the lung is considered to be the major factor, because these oral pathogens might cause pneumonia directly. The initial accumulation of the dental plaque further facilitates the colonization of the upper airway by pulmonary pathogens. A number of epidemiological studies have indicated an independent association between chronic periodontitis and COPD. Some cross-sectional and case-control studies reported significant associations between chronic periodontitis and a history of COPD and lung function decline. Furthermore, periodontitis has been related to the longitudinal decline in the spirometric lung volumes.

If periodontitis is causally associated with a decline of lung function, it is reasonable to implement the periodontal prevention and treatment as therapeutic options to correct the lung malfunction, or at least to support the dedicated treatments of pulmonary diseases, and the common risk factor may explain the association between periodontitis and COPD. The present article briefly reviews the epidemic evidence and common risks between chronic periodontitis and COPD.
\end{abstract}

\section{Introduction}

Periodontitis is an inflammatory reaction to bacterial infections that results in the destruction of the tooth-supporting structures [1]. It is characterized by the interaction between mixed anaerobic, such as
Gram-negative bacteria and the host inflammatory response which results in tissue destruction and tooth loss [2]. In the past two decades, many researchers have provided an increasing body of scientific evidence suggesting that moderately untreated periodontitis are associated with systemic health conditions such as cardiovascular disease, diabetes, and pre-term low birth weight [3]. These diseases are widely prevalent and are associated with an overall burden of the human health.

Chronic obstructive pulmonary disease (COPD) is one of the most common and costly respiratory diseases. COPD is characterized by a slower progressive and irreversible airflow obstruction and the loss of lung tissue which leads to emphysema and tissue remodeling (fibrosis), both of which contribute to a further lung function decline and high mortality [4-6]. In COPD patients, a fixed airflow limitation often results in symptoms such as dyspnea, cough, and sputum production. It is currently the fourth leading cause of death worldwide and is projected to be the third cause of death in the developed countries by 2020 [7]. The association between periodontitis and COPD has been widely investigated. The anatomical continuity between the lungs and the oral cavity makes the latter a potential reservoir of the respiratory pathogens [8]. The exacerbation of COPD was reduced after periodontal treatment [9]. The possible association between periodontitis and COPD was shown in Figure 1.

\section{Epidemiological Studies}

Several studies have revealed an association between lung diseases and poor oral health, such as: 1) Alveolar bone injury; 2) Periodontal attachment loss; 3) Oral hygiene index; and 4) Oral plaque colonization [10-14].

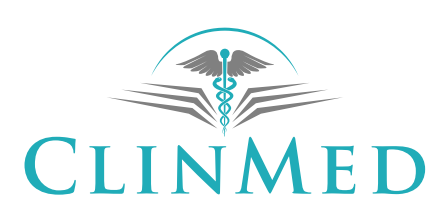

INTERNATIONAL LIBRARY 


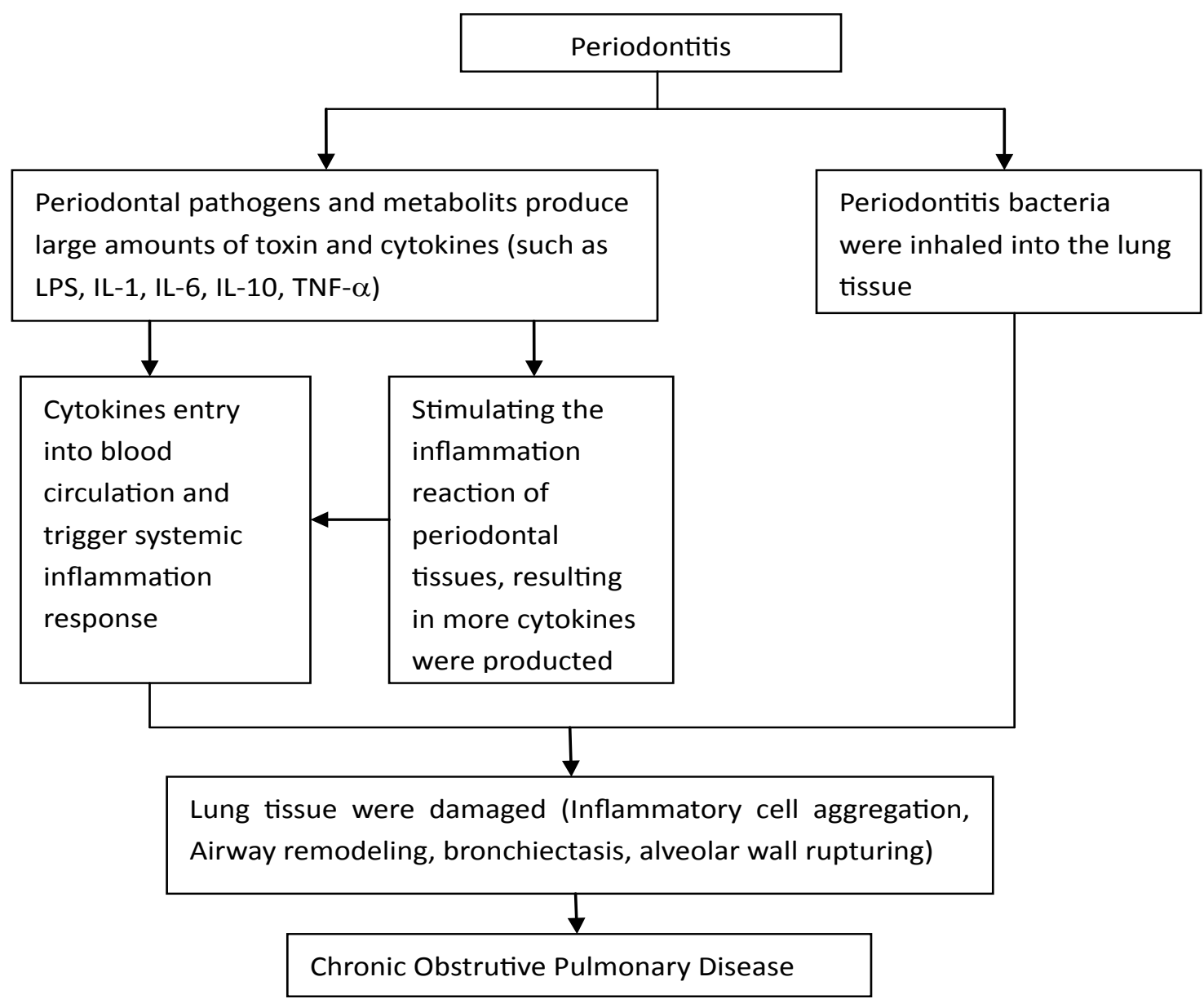

Figure 1: How periodontitis could contribute to the development of COPD.

\section{Case-control study}

Meric, et al. found that compared to the control group, the frequency of exacerbation of COPD was reduced the periodontal treatment group [15]. Takeshi Terashima found that COPD group had fewer remaining teeth, Bleeding on Probing, probing depth, and lower serum albumin levels. COPD was an independent risk factor for poor periodontal health [16]. One case-control study reported a significant association between periodontitis and airway obstruction [17]. When the oral health behavior variables were adjusted simultaneously, the horizontal tooth brushing method was significantly associated with COPD only among non-smokers, while lower regular supra-gingival scaling retained statistically significant associations with COPD among non-smoker. Poor oral health knowledge was significantly associated with COPD in all subgroups. COPD patients appeared to have a poorer periodontal health status than controls with normal pulmonary function. Promoting oral health knowledge, generalizing the appropriate tooth brushing method, and clinical periodontal health care in COPD patients may help to prevent the progression of COPD [1].

\section{Cross-sectional study}

A cross-sectional study has showed that after full adjustment for potential confounders mean CAL was significantly associated with variables of mobile dynamic and static lung volumes, airflow limitation and hyperinflation $(P<0.05)$. Associations were confirmed for mean probing depth, extent measures of CAL/probing depth and number of missing teeth [18]. Zhou, et al. found that poor periodontal health as reflected by more missing teeth and high plaque index were significantly associated with lower quality of life in COPD patients [19]. Jae Ho Chung, et al. revealed that the number of teeth was significantly lower and Decayed-missing-filled (DMF) tooth index was significantly higher in COPD patients [20]. COPD subjects with periodontitis had more marginal bone loss, fewer teeth and more frequent furcation involvement than the COPD subjects without periodontitis [16]. And the severity of periodontitis is positively-associated with inpatient days of patient with respiratory disease [21]. Recently, a lot of epidemiological studies have revealed an association between poor oral health and chronic pulmonary disease. Higher levels of mean CAL were related to lower levels of FVC and FEV1 and higher levels of RV/TLC ratio, subjects with missing teeth had lower absolute values for FEV1, FVC, FEV1/FVC [18,22]. Three cross-sectional epidemiological studies suggested an association between poor oral health (including oral hygiene index, alveolar bone loss, and periodontal attachment loss) and chronic pulmonary disease [10].

\section{Longitudinal (cohort) study}

Hayes, et al. found that for every $20 \%$ increase in 
alveolar bone loss, there was a $60 \%$ increase in risk of COPD [10]. Meric Kucukcoskun revealed that initial periodontitis therapy in COPD patients with CP may decrease the exacerbation frequency [9].

\section{Randomized controlled trial/pilot study}

Zhou found that there were significant improvements in lung function and a reduction in the frequency of COPD exacerbations as a result of periodontal treatment [23]. Based on a small-scale study, periodontal debridement for chronic periodontitis had no effect on quality of life and illness in patients with COPD [24].

\section{Common Risk Factors}

Although the precise mechanisms are unknown, there are several potential explanations of an association between poor periodontal health status and the severity of COPD. Common pathways may explain the connections between periodontitis and systemic disease. Some mechanisms have been proposed to explain the association between periodontitis and COPD. COPD and periodontitis have a similar pathophysiology, specifically, inflammation of local Connective tissue (CT). Risk factors for periodontitis include: 1) Poor oral hygiene; 2) Diabetes mellitus (DM); 3) Smoking; 4) Low socioeconomic status; 5) Age; and 6) Stress [25]. COPD patients were more likely to: 1) Be older; 2) Be less educated; 3) Exercise regularly; 4) Earn lower income; 5) Be current smokers; and 6) Have higher alcohol intake [20]. In addition to the similarities of pathological tissue destruction alluded to earlier, both periodontitis and COPD share similar risk factor profiles.

\section{Smoking}

Smoking is a well-known significant risk factor in COPD, with around $80 \%$ of patients with the disease being current or previous smokers [26]. It is a well-established risk factor for periodontitis [27]. Smoking tobacco has a destructive effect on the periodontitis aggravation [28]. One of the most significant independent risk factors for periodontitis that can affect the host immune-inflammatory response is tobacco smoking. Numerous studies investigating the effects of smoking on IL-1 $\beta$ in saliva or GCF, the findings are conflicting with several suggesting elevated levels, some implying decreased levels and others finding no difference [29]. Continuous exposure to cigarette smokes itself causes deleterious effects on the host that can trigger the development of chronic respiratory diseases, such as chronic obstructive pulmonary diseases (COPD) and lung cancer. COPD is an irreversible airflow obstruction associate with emphysema, fibrosis mucus hypersecretion and persistent colonization of the lower airways by opportunistic [6].

\section{BMI}

There was a significant association between BMI and severity of periodontitis. Berne Eriksson found that compared to current smokers, significantly higher BMI was found among ex-smokers. Higher BMI was found among subjects with diabetes, asthma and respiratory symptoms except chronic productive cough. Mean BMI was slightly but significantly lower in subjects with COPD [30,31].

\section{Old age}

Periodontitis is a chronic disease, the effect of which is accumulated with age. It is therefore reasonable to assume that uncontrolled periodontitis through life would have a greater impact on older patients compared with younger patients. It is known that teeth with periodontal attachment loss have a higher probability of being lost in the future [32]. Prevalence and severity of periodontitis are increased with age. Papapanou and Wennstrom demonstrated that the mean annual rate of dental bone loss was $0.07 \mathrm{~mm}$ and $0.28 \mathrm{~mm}$ in 25-yearold and 70-year-old individuals respectively [33].

COPD is particularly prevalent in the elderly, especially those in institutions such as nursing homes, and in those with several important risk factors. COPD is also associated with age, with lung function declining form early adulthood. The oral cavity is a reservoir for pulmonary infection in nursing home residents, as the oral cavities of older adult residents of nursing homes are more frequently found to harbor respiratory pathogens than those of ambulatory patients. There is some evidence that periodontitis may be associated with risk for pneumonia in community-dwelling older adult patients [34]. Pneumonia causes significant morbidity and mortality, and Chronic obstructive pulmonary disease (COPD) is a known risk factor [35]. Pneumonia risk factors that are modifiable should be considered when attempting to optimize COPD management [36]. A study of 80-years-old Japanese adults found that the adjusted mortality as a result of pneumonia was 3.9 times higher in persons with 10 or more teeth with a probing depth exceeding $4 \mathrm{~mm}$ (i.e. with periodontal pockets) than in those without periodontal pockets [37].

\section{Sex}

Typically, there is also an association with sex, although previously their mainly reflected smoking and working habits. However, in recent years, the incidence has risen in females, which reflects increased smoking habits and leads to a more even sex distribution of the disease. Men who reported being angry had a $43 \%$ higher risk of periodontitis than those who did not [38]. The depth of periodontal pockets was significantly associated with male sex [39].

\section{Bacterial colonization}

According to Scannapieco and Mylotte [17], microorganisms can contaminate the lower airways by four possible routes: 1) Aspiration of oropharyngeal contents; 2) Inhalation of infectious aerosols; 3) Spread of infection from contiguous sites, and 4) Haematogenous spread 
from extrapulmonary sites of infection (e.g. translocation from the gastrointestinal tract).

Microorganisms encompassing the human respiratory microbiota are highly adapted to the host and examples of co-evolution have been described for several human restricted opportunistic pathogens [40]. Periodontitis is a common oral infectious disease that is associated with poor oral hygiene among the general population. The oral cavity provides an optimal environment for bacterial growth because of its suitable humidity and temperature. $1 \mathrm{~mm}^{3}$ of plaque contains $>10^{6}$ bacteria with 300 different anaerobic and facultative anaerobic species [41]. The implication of periodontal bacteria in the initiation and progression of extra-oral systemic diseases has over the last $10-20$ years attracted much attention. Periodontal bacteria can cross epithelial cells by a transcellular mechanism and enter the circulation through physical perturbation of the gingival, they can activate the inflammatory and immune response in multiple ways $[42,43]$. Periodontal bacteria can cross epithelial cells, enter the circulation, invade endothelial cells, induce endothelial cell dysfunction and activate inflammatory and immune responses [44].

Lung inflammation may be affected by aspiration of endotal plaque, which can harbor respiratory pathogens such as Staphylococcus aureus, Acinetobater spp, and Candida albicans. These organisms can be indistinguishable from isolates recovered from tracheobronchial sites in the same patient, NHANES data suggested an association between PD and COPD, which was confirmed by Leuckfeld, et al. [12,16,45,46]. The immune response can stimulate formation, maturation and exacerbation of atheromas. It is clear that oral micro-organisms can cause lung infection and severe PD is associated with pneumonia [47].

It is characterized by inflammation of the periodontium induced subgingival plaque bacteria such as anaerobic gram-negative rods, that can also be associated with COPD exacerbation. Lung inflammation may be affected by aspiration of dental plaque, which can harbor respiratory pathogens such as Staphylococcus aureus, Pseudomonas aeruginosa, Acinetobacter spp and Candida albicans [45]. The most common route by which the oral cavity may influence pulmonary function is the aspiration of harbouring oral bacteria into the lung. When aspirated, oral pathogens cause local damage to the small airways $[45,48]$. Periodontitis may be linked to COPD through microbial species, either by supporting colonization of respiratory pathogens in dental plaque, or by periodontal. Brook's study found elevated antibody levels against Fusobacterium nucleatum and prevotella intermedia, two important periodontal pathogens, in the sputum of patients with an acute exacerbation of chronic bronchitis [49].

\section{Chronic inflammation}

Periodontitis are multi-factorial, resulting from the in- terplay between microbial plaque and the host, driving an increased host immune-inflammatory response. Michele. J. Josey found that fibrinogen level was associated with an increase of mean PD and CAL. With the increase of WBC level, there was a mean increase of mean PD and CAL [50]. The concentrations of pro-inflammatory biochemical mediators, such as cytokines and prostaglandins are measurably increased within the periodontal tissues and the gingival crevicular fluid. These mediators enter the systemic circulation where they may influence other organs, such as the liver, to initiate the acute phase response. This response would then influence atherosclerosis within the endothelium. In addition, bacteria from the gingival crevice may enter the systemic circulation, thus directly affecting [51]. Local pro-inflammatory cytokines are largely responsible for changes which occur. These mediators, which may be found in GCF, saliva and serum, might therefore serve as potential diagnostic or prognostic markers for the progression of periodontitis. IL-1 $\beta$ has been observed in both gingivitis and untreated periodontitis, while treatment of periodontitis results in a dramatic decrease in GCF levels [52]. It is increasingly recognized that periodontitis is associated with a systemic inflammation, with a decrease in serum C-reactive protein (CRP) levels after the treatment of periodontitis [53]. A prospective cohort study found that levels of serum IL-4 and IL-7 were significantly higher in Normal-IgG titer group than in high-IgG titer group, whereas the other Cytokines did not significantly differ between them [54]. IL-6 has also been implicated in periodontitis as it activates osteoclast formation, facilitates bone resorption and T-cell differentiation. IL-8 is involved in the selective recruitment and activation of neutrophils, but alterations of its levels during the pathogenesis of periodontal. Birte Holtfreter found that fibrinogen and hs-CRP were considered as intermediates linking periodontitis with lung function, pulmonary variables showing significant associations with mean CAL [18].

COPD inflammatory status may be modified by dental plaque and/or hematogenous dissemination of inflammatory mediators and plaque microorganism from periodontal pockets [47]. Oztekin, et al. also showed that serum and gingival crevicular fluid high-sensitive C-reactive protein levels were significantly higher in patients with COPD than in controls [55]. Neutrophils also play an important role in both COPD and periodontitis, as their proteases and reactive oxygen species can promote inflammation and destroy CT [56].

\section{Conclusion}

There are a lot of common risk factors between periodontitis and COPD. The treatment of periodontitis (either by repeated prescription of antibiotics or by clinical interventions) might be another way to reduce the incidence of respiratory disease. Nonetheless, a causal association has not been proven, and more studies, in particular intervention studies, are needed. 


\section{References}

1. Zuomin Wang, Xuan Zhou, Jing Zhang, Zhang L, Song Y, et al. (2009) Periodontal health, oral health behaviours, and chronic obstructive pulmonary disease. J Clin Periodontol 36: 750-755.

2. Aarati Nayak, Anita Raikar, Soumya Kambali, Ramakant Nayak, Sumedha Shree, et al. (2016) Histochemical detection and comparison of apoptotic cells in the gingival epithelium using hematoxylin and eosin and methyl green-pyronin: A pilot study. J Indian Soc periodontol 20: 294-298.

3. Williams RC, Offenbacher S (2000) Periodontal medicine: The emergence of a new branch of periodontology. Periodontol 2000 23: 9-12.

4. Taylor JD (2010) COPD and the response of the lung to tobacco smoke exposure. Pulm Pharmacol Ther 23: 376383.

5. William MacNee (2005) Pathogenesis of chronic obstructive pulmonary disease. Proc Am Thorac Soc 2: 258-266.

6. Garmendia J, Morey P, Bengoechea JA (2012) Impact of cigarette smoke exposure on host-bacterial pathogen interactions. Eur Respir J 39: 467-477.

7. Vestbo J, Hurd SS, Agustí AG, Jones PW, Vogelmeier C et al. (2013) Global strategy for the diagnosis, management and prevention of chronic obstructive pulmonary disease. Am J Respir Crit Care Med 187: 347-365.

8. Bansal M, Khatri M, Taneja V (2013) Potential role of periodontal infection in respiratory diseases-a review. J Med Life 6: 244-248.

9. Kucukcoskun M, Baser U, Oztekin G, Kiyan E, Yalcin F (2013) Initial periodontal treatment for prevention of chronic obstructive pulmonary disease exacerbations. J Periodontol 84: 863-870.

10. Hayes C, Sparrow D, Cohen M, Vokonas PS, Garcia RI (1998) The association between alveolar bone loss and pulmonary function: The VA dental longitudinal study. Ann Periodontol 3: 257-261.

11. Russell SL, Boylan RJ, Kaslick RS, Scannapieco FA, Katz RV (1999) Respiratory pathogen colonization of the dental plaque of institutionalized elders. Spec Care Dentist 19: 128-134.

12. Scannapieco FA, Ho AW (2001) Potential associations between chronic respiratory disease and periodontal disease: Analysis of National Health and Nutrition Examination Survey III. J Periodontol 72: 50-56.

13. Scannapieco FA (1999) Role of oral bacteria in respiratory infection. J Periodontol 70: 793-802.

14. Scannapieco FA, Genco RJ (1999) Association of periodontal infections with atheriosclerotic and pulmonary diseases. J Periodontal Res 34: 340-350.

15. Terashima T, Chubachi S, Matsuzaki T, Nakajima T, Satoh $M$, et al. (2017) The association between dental health and nutritional status in chronic obstructive pulmonary disease. Chron Respir Dis 14: 334-341.

16. Leuckfeld I, Obregon-Whittle MV, Lund MB, Geiran O, Bjørtuft $\varnothing$, et al. (2008) Severe chronic obstructive pulmonary disease: Association with marginal bone loss in periodontitis. Respir Med 102: 488-494.

17. Scannapieco FA, Mylotte JM (1996) Relationships between periodontal disease and bacterial pneumonia. J Periodontol 67: 1114-1122.
18. Holtfreter B, Richter S, Kocher T, Dörr M, Völzke H, et al. (2013) Perodontitis is related to lung volumes and airflow limitation: A cross-sectional study. Eur Respir J 42: 15241535.

19. Zhou X, Wang Z, Song Y, Zhang J, Wang C (2011) Periodontal health and quality of life in patients with chronic obstructive pulmonary disease. Respiratory Medicine 105: 67-73.

20. Chung JH, Hwang HJ, Kim SH, Kim TH (2016) Associations between periodontitis and chronic obstructive pulmonary disease: The 2010 to 2012 Korean National Health and Nutrition Examination Survey. J Periodontol 87: 864-871.

21. Ferandez-Plata $R$, Oledo-Torres $D$, Martinez-Briseno $D$, González-Cruz H, Casa-Medina G, et al. (2017) Inpatients days in patients with respiratory diseases and periodontal disease. Gac Med Mex 153: 31-35.

22. Christian Henke, Stephan Budweiser, Rudolf A Jorres (2016) Lung function and associatioin is with multiple dimensions of dental health: A prospective observational cross-sectional study. BMC Res Notes 9: 274.

23. Zhou X, Han J, Liu Z, Song Y, Wang Z, et al. (2014) Effects of periodontal treatment on lung function and exacerbation frequency in patients with chronic obstructive pulmonary disease and chronic periodontitis: A 2-year pilot randomized controlled trial. J Clin Periodontol 41: 564-572.

24. Agado BE, Crawford B, DeLaRosa J, Bowen DM, Peterson $T$, et al. (2012) Effects of periodontal instrumentation on quality of life and illness in patients with chronic obstructive pulmonary disease: A pilot study. J Dent Hyg 86: 204-214.

25. Garcia RI, Nunn ME, Vokonas PS (2001) Epidemiologic associations between periodontal disease and chronic obstructive pulmonary disease. Ann Periodontol 6: 71-77.

26. Mannino DM, Watt G, Hole D, Gillis C, Hart C, et al. (2006) The natural history of chronic obstructive pulmonary disease. Eur Respir J 27: 627-643.

27. Kubota M, Tanno-Nakanishi M, Yamada S, Okuda K, Ishihara K (2011) Effect of smoking on subgingival microflora of patients with periodontitis in Japan. BMC Oral Health 11: 1.

28. Zini A, Sgan-Cohen HD, Marcenes W (2011) Socio-economic position, smoking, and plaque: A pathway to severe chronic periodontitis. J Clin Periodontol 38: 229-235.

29. Wadia R, Booth V, Yap HF, Moyes DL (2016) A pilot study of the gingival response when smokers switch from smoking to vaping. Br Dent J 221: 722-726.

30. Eriksson B, Backman H, Bossios A, Bjerg A, Hedman L, et al. (2016) Only severe COPD is associated with being underweight: Results from a population survey. ERJ Open Res 2.

31. Palle AR, Reddy CM, Shankar BS, Gelli V, Sudhakar J, et al. (2013) Association between obesity and chronic periodontitis: A cross-sectional study. J Contemp Dent Pract 14: 168-173.

32. Beck JD, Sharp T, Koch GG, Offenbacher S (1997) A 5-year study of attachment loss and tooth loss in community-dwelling older adults. J Periodontal Res 32: 516-523.

33. Papapanou PN, Wennström JL, Gröndahl K (1989) A 10year retrospective study of periodontal disease progression. J Clin Periodontol 16: 403-411.

34. Scannapieco FA, Cantos A (2016) Oral inflammation and infection, and chronic medical diseases: Implications for the elderly. Periodontol 2000 72: 153-175. 
35. Jackson ML, Neuzil KM, Thompson WW, Shay DK, Yu O, et al. (2004) The burden of community-acquired pneumonia in seniors: Results of a population-based study. Clin Infect Dis 39: 1642-1650.

36. Crim C, Dransfield MT, Bourbeau J, Jones PW, Hanania NA, et al. (2015) Pneumonia risk with inhaled fluticasone furoate and vilanterol compared with vilanterol alone in patients with COPD. Ann Am Thorac Soc 12: 27-34.

37. Awano S, Ansai T, Takata Y, Soh I, Akifusa S, et al. (2008) Oral health and mortality risk from pneumonia in the elderly. J Dent Res 87: 334-339.

38. Merchant AT, Pitiphat W, Ahmed B, Kawachi I, Joshipura K (2003) A prospective study of social support, anger expression and risk of periodontitis in men. J Am Dent Assoc 134: 1591-1596.

39. Chrysanthakopoulos NA, Chrysanthakopoulos PA (2016) Association between indices of clinically-defined periodontitis and self-reported history of systemic medical conditions. J Investig Clin Dent 7: 27-36.

40. Voges M, Bachmann V, Kammerer R, Gophna U, Hauck CR (2010) CEACAM1 recognition by bacterial pathogens is species-specific. BMC Microbiol 10: 117.

41. Slots J, Rams TE, Listgarten MA (1988) Yeasts, enteric rods and pseudomonads in the subgingival flora of severe adult periodontitis. Oral Microbiol Immunol 3: 47-52.

42. Takeuchi H, Furuta N, Morisaki I, Amando A (2011) Exit of intracellular Porphyromonas gingivalis from gingival epithelial cells is mediated by endocytic recycling pathway. Cell Microbiol 13: 677-691.

43. Olsen I (2008) Update on bacteraemia related to dental procedures. Transfus Apher 39: 173-178.

44. Olsen I (2015) From the acta prize lecture 2014: The periodontal-systemic connectioin seen from a microbiological standpoint. Acta Odontol Scand 73: 563-568.

45. Scannapieco FA, Stewart EM, Mylotte JM (1992) Colonization of dental plaque by respiratory pathogens in medical intensive care patients. Crit Care Med 20: 740-745.

46. Scannapieco FA, Papandonatos GD, Dunford RG (1998) Associations between oral conditions and respiratory dis- ease in a national sample survey population. Ann Periodontol 3: 251-256.

47. Linden GJ, Lyons A, Scannapieco FA (2013) Periodontal systemic associations: Review of the evidence. J Periodontol 84: S8-S19.

48. Sinclair DG, Evans TW (1994) Nosocomial pneumonia in the intensive care unit. $\mathrm{Br} \mathrm{J}$ Hosp Med 51: 177-180.

49. Brook I, Frazier HE (2003) Immune response to fusobacterium nucleatum and prevotella intermedia in the sputum of patients with acute exacerbation of chronic bronchitis. Chest 124: 832-833.

50. Michele J Josey, Anwar T Merchant (2016) Low-grade systemic inflammation may increase the risk of periodontitis. $J$ Evid Based Dent Pract 16: 251-253.

51. Herzberg MC, Nobbs A, Tao L, Kilic A, Beckman E, et al. (2005) Oral streptococci and cardiovascular disease: Searching for the platelet aggregation-associated protein gene and mechanisms of Streptococcus sanguisinduced thrombosis. J Periodontol 76: 2101-2105.

52. Masada MP, Persson R, Kenney JS, Lee SW, Page RC, et al. (1990) Measurement of intreleukin-1a and-1b in gingival crevicular fluid: Implications for the pathogenesis of periodontal disease. J Periodontal Res 25: 156-163.

53. D'Aiuto F, Parkar M, Andreou G, Suvan J, Brett PM, et al. (2004) Periodontitis and systemic inflammation: Control of the local infection is associated with a reduction in serum inflammatory markers. J Dent Res 83: 156-160.

54. Takahashi T, Muro S, Tanabe N, Terada K, Kiyokawa H, et al. (2012) Relationship between periodontitis-related antibody and frequent exacerbations in chronic obstructive pulononary disease. PLoS One 7: e40570.

55. Öztekin G, Baser U, Kucukcoskun M, Tanrikulu-Kucuk S, Ademoglu E, et al. (2014) The association between periodontal disease and chronic obstructive pulmonary disease: A case control study. COPD 11: 424-430.

56. Adam KH Usher, Robert A Stockley (2013) The link between chronic periodontitis and COPD: A common role for the neutrophil? BMC Medicine 11: 241. 